\title{
Screening for bladder cancer: the best opportunity to reduce mortality
}

\author{
Yves Fradet, MD, FRCSC
}

\begin{abstract}
Bladder cancer kills more women than cervical cancer and is also a significant cause of mortality in men. Little progress has been made in improving survival in patients with advanced bladder cancer. Two pilot studies using microhematuria screening have shown that screening for bladder cancer results in close to $80 \%$ downstaging, with high-grade cancers being detected before they have invaded the bladder wall. Results of long-term follow-up even suggest a striking reduction in bladder cancer mortality. The main obstacles to screening for bladder cancer may be overcome if a higher-risk population is identified by designing a risk scale for exposure to cigarette smoke and occupational carcinogens, and through genetic testing for susceptibility to cancer and home hematuria screening, which in itself identifies a population with approximately $3 \%$ to $4 \%$ risk of bladder cancer. The feasibility and cost effectiveness of screening for bladder cancer can be significantly improved by incorporating a secondary screening strategy using a more sensitive and specific bladder cancer marker that is currently available, and by limiting urological evaluations to patients who show positive results on one or more of these tests. Bladder cancer is the most costly cancer to treat in the United States and pharmacoeconomic studies suggest that screening for bladder cancer could not only save lives but also reduce costs per year-life saved. A pilot study is underway and the urology community should be very supportive of studies to validate this opportunity.
\end{abstract}

Can Urol Assoc J 2009;3(Suppl4):S180-3

\section{Introduction}

Screening for bladder cancer is not part of health care recommendations in any country but urinalysis and dipstick testing for microhematuria is routinely performed as part of a regular check-up. Several studies have suggested that the presence of microhematuria is rarely investigated due to fears that cystoscopy creates discomfort to the patient. ${ }^{1}$ Even gross hematuria is frequently neglected, particularly in women, and is often treated as an infection without further evaluation. However, gross and even microscopic hematuria are the most frequent symptoms leading to the diagnosis of bladder cancer.
In the academic urology community, much emphasis has been placed on the treatment of muscle-invasive disease, including the role of chemotherapy and novel therapies. However, despite decades of research and discoveries, at least $25 \%$ of patients with bladder cancer at first diagnosis have muscle-invasive disease that is associated with less than $50 \%$ survival. ${ }^{2}$ Moreover, outcomes of patients with metastatic bladder cancer have been largely unchanged since the introduction of chemotherapy with MVAC (methotrexate, vinblastine, doxorubicin and cisplatin). As well, the benefit of combining systemic chemotherapy with surgery in a neoadjuvant or adjuvant setting has, at best, only modestly improved outcomes. Looking at the evolution in the treatment of cervical cancer over the last several decades, little progress was made through emphasis on more aggressive treatment of advanced disease. Rather, a significant decrease in mortality was achieved by the implementation (without confirmation by randomized trials) of screening with a somewhat invasive test, the Pap test, which is at best $50 \%$ sensitive. At present, young women can even benefit from preventive vaccines for cervical cancers.

It is the author's belief that the real opportunity for reducing bladder cancer mortality lies in early detection and screening and that tools are available that are at least as effective as, if not better than, the Pap test or PSA testing for prostate cancer. The burden is on urologists and specialists of the disease to promote the importance of improved bladder cancer screening and the need for funding of appropriate studies.

\section{Rationale for screening for bladder cancer}

Conceptually, several features of bladder cancer make screening an attractive intervention. There are also several factors that limit the effectiveness of cancer screening, including questions regarding survival rate, disease prevalence, screening efficacy and costs. One common argument against the use of screening for bladder cancer is the low prevalence of the disease. However, factors defining higher risks of bladder cancer have long been recognized. Indeed, cigarette smoking accounts for approximately half of bladder cancers diagnosed and occupational exposure to carcinogens 
has long been identified as a risk factor for bladder cancer. ${ }^{3}$ Moreover, bladder cancer is the fourth most common cancer prevalent in men and the ninth most prevalent in females. Compared to cervical cancer, for which screening is widely practised, bladder cancer is significantly more common with 63210 cases versus 10 370, and has a higher mortality than cervical cancer (13 180 deaths vs. 3710 deaths) in the United States. ${ }^{2}$

All patients who die from bladder cancer do so because of metastatic disease and most have muscle-invasive cancers (stage $\geq \mathrm{T} 2$ ). More than $80 \%$ of muscle-invasive cancers are found at initial diagnosis and in patients who do not have a history of prior superficial tumours. ${ }^{4}$ Hence, the rationale for promoting early bladder cancer screening and detection is to diagnose cancers that are destined to become muscle-invasive before they invade. An additional important factor supporting screening is that, unlike prostate cancer for example, bladder cancer is rarely, if ever, found incidentally at autopsy. ${ }^{5,6}$ Thus, individuals who are diagnosed with bladder cancer through screening would not be subject to over-diagnosis. Finally, urine provides an easy and non-invasive access to cancer cells or shed products that can facilitate early detection of bladder cancer.

\section{Evidence for the potential efficacy of screening}

Most of the knowledge regarding bladder cancer screening is based on 2 studies that tested urine with haemoglobin chemical reagent strips. The rationale for using microhematuria screening with a dipstick test is based on the low initial cost and the recognition that most bladder cancers are diagnosed because they induce hematuria. Messing and colleagues screened 1575 men aged 50 and older who were asked to test their urine for 14 consecutive days at the beginning of the study and to repeat this test 9 months later if all tests during the first 14 days were negative. ${ }^{7}$ If a single test was positive ( $\geq$ "trace"), men were asked to undergo an evaluation that included microscopic urinalysis, urine culture, intravenous urography and office cystoscopy. They found $258(16.4 \%)$ with hematuria, of which 21 (8.1\%) were diagnosed with bladder cancer. The grade and stage of the tumours were compared to that of men aged 50 years and older with newly diagnosed bladder cancer from the Minnesota State Tumor Registry in the same year as the study (509 men). They observed an $80 \%$ downstaging, with only $5 \%$ of screened men detected compared to $24 \%$ of non-screened men with muscle-invasive cancer. At 14 years follow-up, no man with screen-detected bladder cancer had died of bladder cancer, whereas $20.4 \%$ of men with bladder cancer detected in the non-screened population of the same year died of bladder cancer. ${ }^{8}$ In a similar study, Britton and colleagues enrolled 2356 men who were asked to perform home hematuria dipstick testing for 10 days. ${ }^{9}$
They found hematuria in $474(20 \%)$ and bladder cancer in 17 of these men (3.5\%). At 7 years follow-up, 3 of 9 patients considered high grade died of bladder cancer. ${ }^{10}$ These studies suggest that bladder cancer may be detected in patients in a screening setting at an earlier stage, instead of waiting for them to later become symptomatic.

There are several concerns in developing a rationale for a screening policy. One important factor relates to the number of false positives. The home hematuria screening test has a false positive rate ranging from $85 \%$ to $95 \%$ based on the 2 studies. These false positives result in invasive, expensive and unnecessary work-up and introduce a significant physical and emotional burden for the patient. Another consideration involves the finding of a population with sufficient disease prevalence that results in a decrease in the number of false positive evaluations. Recent advances in diagnostic testing provide potential significant solutions to these concerns.

\section{Diagnostic advances}

Three urine-based tests have been commercialized for several years. ${ }^{11}$ The NMP22 point-of-care test (Matritech, Newton, MA) has the advantage that it is an office-based test and is ideally suited for screening. Its sensitivity for detecting tumours is approximately only $50 \%$, with a specificity of $70 \%$ to $80 \%$. Urine cytology has long been available. It has a very high specificity when considering only those tests positive for cancer cells. However, the sensitivity is low even for high-grade cancer, where it is between $50 \%$ and $75 \%$ depending on the study. ${ }^{11,12}$ Moreover, when atypia or suspicious samples are considered, false positive rates increase significantly. ${ }^{13}$ Two cell-based tests are currently available. The UroVysion test (Abbott Molecular) detects loss or gain of 4 chromosomes by in situ hybridization with fluorescent probes. The test is complex and costly. Studies have, however, reported a high specificity but sensitivities varied significantly, from as low as $20 \%$ to as high as $80 \% .{ }^{14}$ ImmunoCyt (SCIMEDX Corporation, Denville, $\mathrm{NJ}$ ) is also a fluorescent test using a cocktail of monoclonal antibodies against 2 antigens. The test is performed on a cytology slide and therefore can provide a cytology result in combination with marker results. ${ }^{15,16}$ ImmunoCyt is less specific than cytology or UroVysion but its sensitivity is the highest among all tests. A recent comparative study of UroVysion and ImmunoCyt in 100 consecutive cases showed a low sensitivity of $13 \%$ for cytology and UroVysion compared to a $73 \%$ sensitivity for ImmunoCyt. ${ }^{17}$

A study of 189 consecutive patients with asymptomatic microhematuria compared ImmunoCyt testing to complete urological evaluation. ${ }^{18}$ ImmunoCyt was positive in 7 out of 8 bladder tumours detected ( $87 \%$ sensitivity) and had $91 \%$ specificity. If assessment of these patients had been 
based on ImmunoCyt testing alone, 154 costly and invasive diagnostic procedures could have been avoided and only one small low-malignant potential tumour would have been missed. ImmunoCyt and cytology were also used to screen aluminum workers in the United States, Brazil and Germany who participated on a voluntary basis under the supervision of epidemiologists. The preliminary analysis of 4494 workers screened in the first year detected 6 cancers. Cytology detected 3 out of 6 and ImmunoCyt detected 6 out of $6(100 \%$ sensitivity). The specificity of both tests was $99 \%$ in this cohort of asymptomatic men. Hemstreet and colleagues used 1 of the 2 markers of the ImmunoCyt test to screen a cohort of 1788 exposed and 373 non-exposed workers in China. ${ }^{19}$ This single marker showed a $56 \%$ sensitivity and a $98 \%$ specificity. Interestingly, in patients with a positive test and negative cystoscopy, the risk of subsequent finding of cancer 6 months later was 38 times greater than for workers with negative urine test (Cl 17-85). The combination of ImmunoCyt and cytology in several studies showed more than 95\% sensitivity in detecting high-grade cancers at the non-invasive stage of carcinoma in situ, which are the primary target in screening strategies, since they are more likely to lead to muscle-invasive cancer.

An optimal strategy for bladder cancer screening remains to be determined. Home hematuria screening during 10 to 15 days on a yearly basis would be inexpensive as a primary tool; however, it would be very costly if all patients who tested positive received a full urological evaluation. Using a sensitive and specific non-invasive urine test as a secondary screening tool would be more easily accepted by patients and primary physicians and would lead to significantly fewer unnecessary investigations by cystoscopy. This strategy is currently being tested in a screening study funded through the MD Anderson Specialized Programs of Research Excellence (SPORE), in which subjects use multiple Hemastix tests and patients with positive Hemastix tests undergo cystoscopy along with 3 marker tests; NMP22, UroVysion and ImmunoCyt. Several centres, including this one, are involved in this pilot study, which could lead to a short-term recommendation for the investigation of microhematuria as well as a design of a larger-scale screening trial.

\section{Identification of higher-risk populations}

The ability to identify populations at higher risk of developing urothelial carcinoma would be helpful. This could be done in part by refining our understanding of age, cigarette smoking and occupational exposure and by developing a model for risk assessment. Genetic analyses provide yet another approach to identifying individuals more susceptible to the development of bladder cancer under exposure to these carcinogens. Moore and colleagues adequately addressed this issue by studying blood samples from a Spanish case-control study that tested the hypothesis that hypomethylation of DNA from white blood cells was more frequently observed in patients with urothelial carcinoma. ${ }^{20}$ The cytosine methylation content in leukocyte DNA of 775 patients with bladder cancer (cases) and 397 controls were analyzed by high-performance capillary electrophoresis and Hpall digestion. The median percentage of cytosine methylation DNA was significantly lower in cases than in controls. Moreover, investigators were able to stratify patients in groups at increasing risk of cancer on the basis of their smoking status and level of methylation. Non-smokers with the highest level of methylation had the lowest risk of bladder cancer, whereas current smokers with hypomethylation had a 25.5 times higher risk of cancer. A number of other genetic markers are under investigation and may provide improved methods to identify the appropriate population for bladder cancer screening.

\section{Cost-effectiveness}

Bladder cancer is the most costly cancer per patient (from diagnosis to death) in the United States. ${ }^{21,22}$ A recent study using a Markov model to estimate the cumulative cancerrelated cost and efficacy of screening versus no screening showed that urine-based markers are cost-effective in a high-risk population. ${ }^{23}$ Indeed, screening is the most costeffective strategy when cancer incidence in the population is $>1.6 \%$ if tumour markers cost less than $\$ 126$ and have a sensitivity of $>26 \%$ and specificity $>54 \%$, assuming a downstaging with screening of $20 \%+$ and office cystoscopy costs less than $\$ 694$. Obviously, if downstaging is closer to the $70 \%$ to $80 \%$ observed in the 2 small available studies, it would have a significant impact on cost savings. Equally effective would be the selection of a target population at higher risk and the use of more specific tests. For example: at $4 \%$ cancer incidence, which is the incidence observed in individuals testing positive in hematuria screening, a tumour marker specificity of $86 \%$ yields a cost per life-year savings of $\$ 101,000$. A decrease in specificity to $50 \%$ would result in a discounted cost per life-year saved of $\$ 4,384$ and a specificity of $30 \%$ would increase this cost to $\$ 25,599$ per life-year saved. By comparison, the cost per life-year saved for breast cancer patients screened by mammography varies from $\$ 16,000$ to $\$ 19,000$, and for cervical cancer it is estimated to be $\$ 80,000$. The cost per life-year saved for prostate cancer is estimated to be between $\$ 2300$ and $\$ 5000$.

\section{Conclusion}

In summary, bladder cancer is frequent and an important cause of mortality that could potentially be prevented. Improved use of new urine-based tumour markers should 
be emphasized, since they could represent an immediate, efficient screening tool in patients already identified at higher risk due to the presence of microhematuria during routine urinalysis. The identification of high-risk cancer at a nonmuscle-invasive stage coupled with improvements in intravesical therapy (that will hopefully result from ongoing research and discovery) would not only reduce mortality but also morbidity as well as costs associated with the treatment of more advanced disease. Improvements in fluorescent cystoscopy also enable the detection of non-visible cancers more effectively in patients with seemingly falsepositive tests. ${ }^{24}$ Altogether, these observations suggest that the opportunity provided by bladder cancer screening with these new strategies could be at least as efficient as the significant advancements achieved by Pap test screening for cervical cancer. It is the responsibility of the urology community to move the field forward in this direction.

From the University of Laval, Québec, QC

Competing interests: The author owns stock in, and is Chief Medical Officer at, DiagnoCure Inc.

This paper has been peer-reviewed.

\section{References}

1. Nieder AM, Lotan Y, Nuss GR, et al. Are patients with hematuria appropriately referred to Urology? A multi-institutional questionnaire based survey. 2009. In press.

2. Jemal A, Murray T, Ward E, et al. Cancer statistics, 2005. CA Cancer I Clin 2005;55:10-30.

3. Silverman DT, Hartge P, Morrison AS, et al. Epidemiology of bladder cancer. Hematol Oncol Clin North Am 1992;6:1-30

4. Vaidya A, Soloway M, Hawke C, et al. De novo muscle invasive bladder cancer: is there a change in trend? J Urol 2001;165:47-50; discussion 50.

5. Marshall VF. Current clinical problems regarding bladder tumors. Cancer 1956;9:543-50.

6. Ressequie $L$, Nobrega FT, Farrow GM, et al. Epidemiology of renal and ureteral cancer in Rochester, Minnesota, 1950-1974, with special reference to clinical and pathologic features. Mayo Clin Proc. 1978:53:503-10.
7. Messing $E M$, Young $T B$, Hunt VB, et al. Comparison of bladder cancer outcome in men undergoing hematuria home screening versus those with standard clinical presentations. Urology 1995;45:387-96; discussion 396-7.

8. Messing EM, Madeb R, Young T, et al. Long-term outcome of hematuria home screening for bladder cancer in men. Cancer 2006;107:2173-79.

9. Britton JP, Dowell AC, Whelan P, et al. A community study of bladder cancer screening by the detection of occult urinary bleeding. J Urol 1992;148:788-90.

10. Mayfield MP, Whelan P. Bladder tumours detected on screening: results at 7 years. Br J Urol 1998;82: 825-8.

11. Lotan Y, Roehrborn CG. Sensitivity and specificity of commonly available bladder tumor markers versus cytology: results of a comprehensive literature review and metaanalyses. Urology 2003;61:109-18.

12. Raitanen MP, Aine R, Rintala $E$, et al. Differences between local and review urinary cytology in diagnosis of bladder cancer. An interobserver multicenter analysis. Eur Urol 202;41:284-89.

13. Nabi $G$, Greene $D R, O^{\prime}$ Donnell M. How important is urinary cytology in the diagnosis of urological malignancies? Eur Urol 2003;43:632-6.

14. Lodde $M$, Fradet $Y$. The detection of genetic markers of bladder cancer in urine and serum. Curr Opin Urol 2008;18:499-503.

15. Mian C, Maier K, Comploj E, et al. uCyt+/ImmunoCyt ${ }^{T M}$ in the detection of recurrent urothelial carcinoma. An update on 1991 analyses. Cancer (Cancer Cytopathol) 2006;108:60-5.

16. Lodde $M$, Mian C, Wiener $H$, et al. Detection of upper urinary tract transitional cell carcinoma with ImmunoCyt: a preliminary report. Urology 2001;58:362-6.

17. Sullivan PS, Nooraie F, Sanchez H, et al. Comparison of ImmunoCyt ${ }^{\top M}$, UroVysion ${ }^{\top M}$, and urine cytology in detection of recurrent urothelial carcinoma: a "split-sample" study. Cancer (Cancer Cytology) 2009. In press.

18. Schmitz-Dräger $B J$, Beiche $B$, Tirsar $L A$, et al. Immunocytology in the assessment of patients with asymptomatic microhaematuria. Eur Urol 2007;51:1582-8.

19. Hemstreet III GP, Yin S, Ma Z, et al. Biomarker risk assessment and bladder cancer detection in a cohort exposed to benzidine. J Natl Cancer Inst 2001;93:427-36.

20. Moore LE, Pfeiffer RM, Poscablo C, et al. Genomic DNA hypomethylation as a biomarker for bladder cancer susceptibility in the Spanish Bladder Cancer Study: a case-control study. The Lancet Oncol 2008:9:359-66.

21. Urologic Diseases in America 2007. Editors MS Litwin, CS Saigal. National Institute of Diabetes and Digestive and Kidney Diseases.

22. Botteman MF, Pashos CL, Redaelli A, et al. The health economics of bladder cancer : a comprehensive review of the published literature. Pharmacoeconomics. 2003;21:1315-30.

23. Lotan Y, Svatek RS, Sagalowsky Al. Should we screen for bladder cancer in a high-risk population? A cost per life-year saved analysis. Cancer 2006;107:982-90.

24. Fradet $Y$, Grossman HB, Gomella $L$, et al. A comparison of hemaminolevulinate (HKL) fluorescence cystoscopy and white light cystoscopy for the detection of carcinoma in situ in patients with bladder cancer. J Urol 2007;178:68-73.

Correspondence: Dr. Yves Fradet, University of Laval Cancer Research Centre, CHUQ - Hôtel-Dieu de Québec, 11 côte du Palais, Québec, QC GIR 2J6 ; fax 418-691-5562; wves.fradet@crhdq.ulaval.ca 